\title{
La construction d'un marché européen des eaux embouteillées. Enjeux, acteurs et déroulement des négociations de la directive 80/777 sur les eaux minérales (années 1950-années 1980)
}

\author{
Nicolas MARTY
}

L'eau en bouteille s'est diffusée très largement dans le monde depuis la fin des années 1980, à tel point qu'elle est devenue un des produits emblématiques de la société de consommation de masse, mondiale et globalisée. C'est un produit dont l'origine est spécifiquement européenne. Depuis le milieu du XIXe siècle, l'eau embouteillée a connu des succès très importants en Europe occidentale, créant des marchés émergents dans des contextes normatifs différents. Trois modèles de régulation se sont en effet construits avant la Première Guerre mondiale. Le modèle «latin» (France, Espagne, puis Italie et Belgique) où l'intervention classificatoire de l'État s'appuie sur une expertise scientifique avant d'être relayée par les contrôles de l'administration conservant l'aspect strictement thérapeutique des eaux. La mise sur le marché des produits y nécessite une autorisation préalable. Le modèle allemand où les entreprises agissent à travers des accords de branche sur la base d'une vision uniquement chimique du produit (degré de minéralisation). Enfin, un modèle britannique caractérisé par l'absence de définition de la qualité du produit. Un modèle au sein duquel c'est essentiellement au consommateur de faire le meilleur choix dans des produits très divers mis sur le marché (principe du buyer beware). Ces trois modèles se sont maintenus jusqu'à l'après Seconde Guerre mondiale.

En 1980, ces trois modèles furent unifiés par la création d'une norme européenne, précisée dans la directive CE 80/777. L'objectif de cet article est de montrer comment s'est construite concrètement cette norme. Pour cela, il est nécessaire de préciser que cette publication correspond à une partie d'un travail plus large dont les objectifs sont de montrer l'importance des normes dans la construction des marchés des produits industriels à travers l'exemple de l'eau embouteillée. ${ }^{1}$ Il part du principe que l'histoire des produits du secteur des boissons et de l'alimentation offre une contribution fondamentale pour comprendre l'évolution des sociétés vers la consommation de masse. Il se base sur l'idée que les normes et la qualité des produits sont le résultat d'une construction sociale dépendant du contexte général des sociétés et de l'activité d'une

1. Les programmes ANR ECA (Emballages et conditionnements alimentaires, 1850-2000), Universités de Tours et Perpignan, la Maison des sciences de l'Homme d'Aquitaine, le CRH de l'EHESS et Euwol (European Ways of Life, ESF, Programme Inventing Europe), ont été essentiels tant au plan matériel que conceptuel pour la rédaction de cet article. Je remercie vivement Christophe Bouneau, Florence Hachez-Leroy, Marc de Ferrière le Vayer, Jean-Pierre Williot, Ruth Oldenziel, Per Øtsby et Karin Zachmann pour leur aide précieuse dans la recherche menée. 
multiplicité d'acteurs à la fois marchands et non marchands. ${ }^{2}$ Enfin, c'est un travail qui repose sur une approche comparatiste, afin de comprendre les différences qui peuvent naître pour un même type de produit dans différents pays de l'Europe occidentale.

Dès le traité de Rome, l'un des objectifs des Communautés européennes était de favoriser la circulation des biens par l'abaissement des barrières économiques, mais aussi des barrières non tarifaires. Or, parmi celles-ci figuraient les réglementations qui, en fixant les conditions de vente, d'utilisation des produits et plus encore celles de fabrication, pouvaient constituer des distorsions de concurrence. La phase longue et complexe de négociations sur l'harmonisation des législations nécessite que l'on présente au préalable les enjeux et les acteurs qui y participent. Le déroulement des négociations est ensuite évoqué en suivant une chronologie qu'il a été nécessaire de reconstituer pour bien en saisir les principaux tournants. Enfin, la suite de l'adoption de la norme CE mérite qu'on observe son application et ses principales conséquences.

\section{Enjeux et Acteurs}

\section{Des enjeux considérables}

Les enjeux des négociations sur l'harmonisation des normes dans le domaine des eaux embouteillées étaient considérables. D'abord parce qu'il était frappant de constater qu'il n'existait pas, au cours des années 1940-1970, de marché européen des eaux embouteillées, mais seulement une juxtaposition de marchés nationaux. Entre 1950 et le milieu des années 1970, les exportations représentaient en moyenne $5,5 \%$ de la production domestique. ${ }^{3}$ Les différences de régulation étaient un obstacle clair au développement d'un marché européen. Ainsi, les entreprises qui souhaitaient faire entrer en France des eaux minérales devaient-elles se conformer aux prescriptions d'un arrêté interministériel du 20 avril 1959 qui reprenait l'essentiel des éléments de la législation française, très contraignante pour les producteurs français euxmêmes. Il n'était pas rare que certaines entreprises, bien que renommées comme la fameuse Appolinaris allemande, ne reçoivent pas l'autorisation d'importer en France sous l'appellation eau minérale. ${ }^{4}$ De ce fait, pour chaque pays, l'harmonisation était

2. Cf. notamment M. BRUEGEL, A. STANZIANI, Pour une histoire de la qualité alimentaire, in: Revue d'histoire moderne et contemporaine, 3(2004), p.14 et D. SMITH, J. PHILIPPS, Food Policy and regulation: a multiplicity of actors and experts, in: D. SMITH, J. PHILIPPS (dir.), Food, Science, Policy and Regulation in the $20^{\text {th }}$ Century, Routledge, London, 2000, pp.1 et 7.

3. Document GESEM, Le marché des eaux minérales, 1953-1978.

4. CHAN [Centre Historique des Archives Nationales, Fontainebleau] 2000 113, art.32, Note sur l'importation des eaux minérales en France, Direction générale de la Santé (DGS), mai 1971; 20030384 , art.5, DGS, Arrêté de 1959. 
à la fois synonyme de danger comme de potentialités importantes. ${ }^{5}$ Par exemple, si l'harmonisation devait reposer sur les bases de la réglementation allemande, un grand nombre d'eaux minérales françaises, considérées logiquement comme des produits médicamenteux seraient, telles les Heilwässer (eaux médicinales), réduites à n'être diffusées quasi uniquement qu'en pharmacie.

Les différences qui existaient dans la structure des branches étaient aussi lourdes de menaces. Il est bien évident que les organisations professionnelles en étaient bien conscientes, comme le montrent de nombreux courriers conservés dans les archives de la direction générale de la Santé. Ainsi, Paul Bordier, alors président de la Chambre syndicale des eaux minérales de France, expliquait dans une lettre à ses homologues italiens et belges au moment des négociations de 1978 que la directive «est d'une importance capitale par ses conséquences sur nos activités industrielles». ${ }^{6}$

De leur côté, les États n'avaient pas les mêmes appréciations selon qu'ils étaient, ou n'étaient pas producteurs d'eaux embouteillées, si leur réglementation interne était plus ou moins spécifique et homogène, et si leur industrie avait, ou non un réel potentiel d'exportation. Ainsi, les Pays-Bas initialement, puis le Danemark, l'Irlande et le Royaume-Uni après 1973, étaient des acteurs qui n'avaient pas la même motivation dans cette négociation que les pays producteurs. Dans le domaine des eaux minérales, des firmes comme les groupes Perrier et BSN en France, Spa Monopole en Belgique, pouvaient être considérés comme des «champions nationaux» dont les États étaient prêts à défendre les intérêts. ${ }^{7}$ Enfin, les pays non producteurs pouvaient être intéressés à cette négociation dans la mesure où ils avaient aussi à défendre une certaine vision des biens alimentaires, de leur politique de concurrence, ou plus généralement de leurs relations au droit.

\section{Une grande quantité d'acteurs}

Les organisations professionnelles nationales et derrière elles, les entreprises, jouèrent un rôle de premier ordre pendant la négociation. Le contexte était alors celui de l'émergence de l'identité européenne dans nombre de branches et d'industries, qui n'avaient pas connu, contrairement à des activités comme la métallurgie ou l'acier, les tentatives d'entente européenne d'avant-guerre. ${ }^{8}$ C'est en avril 1953 que se forma le Groupement européen des sources et eaux minérales (GESEM) à Paris, sur une initiative franco-al-

5. Nous sommes bien là dans la dialectique relevée par Patrick Fridenson pour le marché automobile: l'Europe comme ressource, l'Europe comme problème. P. FRIDENSON, Stratégies des groupes automobiles et structure du marché en Europe, 1979-1992, in: E. BUSSIÈRE, M. DUMOULIN, S. SCHIRMANN (dir.), Milieux économiques et intégration européenne au XX ${ }^{\mathrm{e}}$ siècle. La relance des années quatre-vingt (1979-1992), CHEF, Paris, 2007, pp.333-348, ici p.333.

6. CHAN, 1987 0334, art.44, DGS, Paul Bordier à Carmelo Callipo, directeur du Federterme (Rome) et Marcel Cats, président de la Fédération Nationale des eaux de boisson de Belgique, 23.05.1978.

7. J.C. DEFRAIGNE, La transition des années 1970 en terme de politique industrielle. Vers l'abandon de la stratégie des champions nationaux, in: É. BUSSIÈRE, M. DUMOULIN, S. SCHIRMANN (dir.), op.cit., pp.225-280, ici pp.228-229.

8. R. RANIERI, Les entreprises et les branches industrielles et leurs modèles, in : É. BUSSIÈRE, M. DUMOULIN, S. SCHIRMANN (dir.), op.cit., pp.163-166. 
lemande. ${ }^{9}$ Le président de la Chambre syndicale française, René Loubet, et celui du Verband Deutscher Mineralbrunnen (VDM), Otto Wuttke, s'étaient rencontrés de manière informelle lors de la première «Conférence européenne des soft-drinks» à Hambourg en octobre 1952. Il s'agissait surtout de travailler ensemble à la promotion de l'eau minérale, d'échanger des renseignements sur les prix et les marchés d'exportation. ${ }^{10}$ Les associations autrichienne, italienne, espagnole et portugaise participèrent au premier congrès du groupement, et René Loubet fut nommé président. ${ }^{11}$ Après plusieurs réunions préparatoires la première assemblée générale eut lieu en mai 1956 à Milan et en octobre 1957, les dirigeants des entreprises des pays membres du Marché commun organisèrent un comité de travail afin de réfléchir aux conséquences potentielles de cette création. Ils décidèrent la constitution d'une association spécifique aux membres de la Communauté afin d'être reconnus comme des interlocuteurs valables par les institutions de la CE: 1'Union européenne des sources d'eaux minérales (Unesem, 1959).

Les institutions européennes jouèrent, elles aussi, un rôle important dans la négociation. La question du rapprochement des dispositions législatives des pays membres de la CEE dans le domaine des produits alimentaires et des boissons fut posée en effet très tôt. ${ }^{12}$ Les premières discussions eurent lieu au sein du sous-comité pour le contrôle sanitaire des denrées alimentaires, réuni à Rome en novembre 1957. ${ }^{13}$ Les chefs de délégation s'étaient consultés au sujet de l'exécution du traité constituant la CEE en matière de produits alimentaires et de boissons. L'unanimité se fit rapidement sur le constat qu'il n'y avait aucune raison de maintenir les divergences des systèmes de protection nationaux de la santé en matière de produits alimentaires et de boisson. Les membres de la délégation virent immédiatement que l'on pourrait utiliser l'article 100 pour proposer des directives portant sur la compilation des dispositions relatives à la désignation, la composition et le mode de préparation de tous les produits alimentaires et boissons afin de les incorporer immédiatement dans les législations nationales. Les membres des délégations savaient bien que le vote du Conseil nécessitait une préparation précise avant de présenter ces législations

9. H. MIARD-DELACROIX, R. HUDEMANN, Les processus de rapprochement franco-allemands de mutation et d'intégration dans les années cinquante, in: H. MIARD-DELACROIX, R. HUDEMANN (dir.), Wandel und Integration: deutsch-französische Annäherungen der fünfziger Jahre, Oldenbourg, München, 2005.

10. R. LOUBET, Der Weg zur Arbeitsgemeinschaft Europäischer Mineralbrunnen, in: Der Naturbrunnen, 9(1954), pp.3-7, ici p.4.

11. Les industriels italiens étaient très favorables à des accords transnationaux entre producteurs, mais hostiles à une autorité supranationale dotée de pouvoirs d'intervention sur les marchés. Cf. F. PETRINI, Les milieux industriels italiens et la création du marché commun, in: Revue d'histoire de l'intégration européenne, 1(2003), pp.11-12.

12. Les historiens évoquent plutôt la mise en place du marché agricole européen comme point de départ la mise au point de normes européennes en matière alimentaire. H.J. TEUTEBERG, Agenda for a comparative European History of Diet, in: H.J. TEUTEBERG (éd.), European Food History: a research Review, Leicester University Press, Leicester, 1992, p.14.

13. AHUE [Archives Historiques de l'Union Européenne], CM2/1958-000077, Note sur le rapprochement des dispositions législatives des pays membres de la CEE dans le domaine des produits alimentaires et des boissons, 09.12.1957. 
harmonisées. Il fut donc constitué un groupe d'experts en matière de santé publique et d'alimentation.

La direction des affaires industrielles de la Commission fut chargée du travail de mise en œuvre. Les services associés étaient: la direction générale de l'Agriculture, la direction du Marché intérieur et du rapprochement des législations et le Service juridique. ${ }^{14} \mathrm{La}$ Commission transmit ensuite sa proposition de directive au Conseil des communautés européennes. La proposition d'une directive fondée sur l'article 100 nécessitant des modifications des dispositions législatives dans certains états membres, le Conseil dut transmettre la directive, pour avis, au Comité économique et social (CES) ainsi qu'au Parlement européen. Au sein du CES, la section économique chargea le groupe d'étude «entraves techniques» de travailler au rapport final. Plusieurs commissions du Parlement européen intervinrent aussi: la commission des affaires sociales, la commission juridique, la commission économique. ${ }^{15}$ Enfin, alors que les négociations étaient difficiles, pendant les années 1970, le comité des représentants permanents (Coreper) tint les premiers rôles.

Le processus d'harmonisation des législations suscita aussi une mobilisation de l'expertise. Celle-ci relevait de plusieurs échelles à la fois nationales et internationales et de plusieurs champs. Les experts étaient concernés scientifiquement, d'abord par les problèmes de bactériologie et d'analyse des eaux et, plus généralement, par tout ce qui touchait à la santé publique; mais ils s'intéressaient aussi aux questions administratives et économiques. La complexité provenait de la diversité des intervenants. Un exemple éloquent en est fourni par la mobilisation administrative que suscitait cette question en France, en 1967. Lors du dépôt du projet par l'Unesem au cours d'une réunion du sous-groupe «boissons sans alcool» de la direction de l'industrie de la commission européenne, étaient saisis: la direction des affaires économiques et financières du ministère des Affaires étrangères, les services des douanes, la DREE, le commerce intérieur et les prix du ministère des Finances; le service de répression des fraudes, la direction de l'industrie agricole et de l'organisation économique, le service des relations internationales du ministère de l'Agriculture; et encore le service des industries chimiques du ministère de l'Industrie; et pour finir, le service des relations internationales et la Direction générale de la santé du ministère de la Santé et des affaires sociales. ${ }^{16}$ À l'échelle européenne, les services de la commission travaillaient avec un groupe de conseillers ad hoc composé de conseillers et d'attachés. Ce groupe fut ensuite institutionnalisé en 1969 avec la création d'un Comité permanent des denrées alimentaires, considéré comme compétent dans le domaine des eaux minérales. D'autres groupes d'experts de niveau européen étaient aussi saisis ou in-

14. AHUE, BAC 03 1974, Projet d'une proposition: directive du conseil relative au rapprochement des législations des états membres concernant l'exploitation et la mis dans le commerce des eaux minérales naturelles.

15. AHUE, CES 3543, Saisine du CES d'une proposition de directive, décembre 1970; POE 1097, Parlement. Rapports de commissions 1970-1971.

16. CHAN, 2003 0384, art.5, DGS, Comité interministériel pour les questions de coopération économique européenne, service du Premier ministre, sur la réunion de la sous commission Boisson sans alcool, 15.11.1967. 
téressés par les négociations, comme le Groupe des experts en nomenclature douanière de la Direction du marché intérieur de la commission des Communautés européennes. ${ }^{17}$

Un processus parallèle, les travaux pour la mise au point du Codex alimentarius compliqua les négociations. L'institution du Codex avait été créée officiellement à l'échelle mondiale en 1962. Ce codex devait aboutir à des normes protégeant la santé des consommateurs et la loyauté des transactions commerciales dans le domaine des denrées alimentaires. Le travail de construction des normes proprement dit était effectué dans des comités s'intéressant soit à des sujets horizontaux (hygiène, étiquetage, méthodes d'analyses), soit à des groupes de produits particuliers (produits laitiers, fruits et légumes tropicaux etc.). Parmi eux, on trouvait celui des eaux minérales, dont la responsabilité incombait à la Suisse. ${ }^{18}$ Ce processus avait été enclenché en dehors des institutions des Communautés européennes.

\section{Le déroulement des négociations}

\section{La préparation des travaux d'harmonisation 1959-1971}

La préparation de l'harmonisation eut d'abord lieu au sein du groupement professionnel européen des eaux minérales. Selon son premier dirigeant, René Loubet, c'est bien «conformément aux directives des hautes autorités du Marché commun à Bruxelles», que le Gesem Unesem entreprit le travail de réflexion sur l'harmonisation des législations nationales, en mai $1957 .{ }^{19}$ Un comité de travail pour l'examen des problèmes posés par le marché commun fut créé lors d'un congrès du Gesem en octobre 1957. Parallèlement, la décision fut prise de prendre part aux travaux d'élaboration de la norme régionale du Codex pour les eaux minérales. Un compromis était trouvé dès 1962 au congrès de Florence du Gesem Unesem. ${ }^{20}$ Celui-ci reposait sur le principe de la coexistence des deux critères de reconnaissance des eaux minérales: propriétés favorables à la santé d'une part, caractéristiques de composition chimique d'autre part. Dans son exposé des motifs, l'Unesem précisait:

"On a retenu pour la définition de l'eau minérale des critères différents selon les pays sur lesquels elle se fonde aujourd'hui. Il a été considéré qu'une eau minérale peut être estimée telle soit parce qu'elle est douée de propriétés thérapeutiques ou simplement favorables à

17. AHUE, BAC 028 - 6, Proposition d'une directive du Conseil. Résumé Aide-mémoire, 15.01.1975; BAC 03 1974 - 8, Résolution du conseil, 13.11.1969, in: Journal Officiel, 19.11.1969; CHAN, 2003 0384, art.5, Note du Groupe des experts en nomenclature douanière, 29.01.1968.

18. J. BIZET, Rapport d'information fait au nom de la délégation du Sénat pour l'Union européenne sur le Codex alimentarius, $\mathrm{n}^{\circ}$ 450, session ordinaire de 1999-2000.

19. CHAN, 2003 0384, art.5, DGS, Rapport de M. Loubet devant le conseil supérieur du thermalisme, 12.04.1962.

20. O. WUTTKE, Der Kongress von Florenz. Auf dem Wege zu einem Einheitlichen europäischen Mineralwasserrecht, in: Der Naturbrunnen, 12(1962), p.212. 
la santé, comme il est exigé en France, Belgique et Italie, soit en raison de sels minéraux dissous ou de gaz carbonique libre qu'elle contient, comme c'est le cas en Allemagne. Qu'une eau minérale naturelle ressortisse d'ailleurs à l'un ou l'autre concept, ses qualités devront être reconnues par une académie de médecine ou, à défaut, par un organisme officiel de compétence équivalente". ${ }^{21}$

Si ce compromis ne fut présenté sous forme de proposition à la CEE qu'en 1965, c'est qu'un problème était posé par la France qui n'acceptait pas l'utilisation de l'eau minérale pour l'embouteillage des soft drinks. Les autorités médicales françaises restaient arc-boutées sur l'interdiction de mélanger l'eau minérale à quelque autre produit. Finalement, la proposition de directive construite par l'Unesem en 1965 proposa sur ce point une dérogation temporaire en faveur de la France. C'est donc en décembre 1965 que les services de la Communauté engagèrent, avec l'assistance d'un groupe d'experts désignés par les gouvernements, l'étude de la directive d'harmonisation. Ce n'est pourtant qu'au début de 1970 que la Commission parvint à proposer un projet. Ce délai important s'explique d'abord par le fait que la CEE connaissait alors une grave crise institutionnelle, qui touchait indirectement les travaux les plus techniques. La directive des eaux minérales était préparée au sein de la Direction générale des affaires industrielles, par les services de «circulation des marchandises» et des «produits agricoles transformés» qui étaient alors accaparés par les négociations agricoles complexes qui se déroulaient dans le même temps.

Mais cette difficulté interne n'explique pas seule le retard de la directive. Dans le même temps, en effet, le comité spécialisé du Codex alimentarius s'était réuni à plusieurs reprises à compter de 1966. Les experts européens l'avaient fait savoir dès 1957, ils préféraient avoir en main les résultats des travaux du Codex pour l'harmonisation des législations. Ces négociations, après quelques péripéties, aboutirent finalement à un accord en 1968 qui rappelait pratiquement sur tous les points le compromis des professionnels de $1965.22 \mathrm{Il}$ est vrai que dans le même temps, les barrières douanières avaient été levées le 1er janvier 1968 et tous les travaux en cours pour supprimer les obstacles techniques et non tarifaires à la circulation des marchandises, et plus particulièrement dans le domaine alimentaire, étaient accélérés. ${ }^{23}$ La proposition de directive sur l'harmonisation des législations en matière d'eaux minérales était prévue dans la quatrième phase du «programme général» du 28 mai 1969, établie en vue de l'élimination des entraves techniques aux échanges. On voit que la Commission n'avait pas attendu la «relance de La Haye» pour tenter d'accélérer les dossiers techniques.

21. CHAN, 2003 0384, art.5, DGS, UNSEM, Exposé des motifs de la proposition de directive d'harmonisation, 04.12.1965.

22. CHAN, 2003 0384, art.5, DGS, Rapport du Dr Fabre au nom de la commission des eaux minérales de l'Académie de médecine, 15.02.1964; Lettre accompagnant le document de travail sur une proposition de directive, 07.10.1969; Compte rendu des travaux du comité Codex à Bad Ragaz, mai 1968.

23. AHUE, CES 3543, Liste des propositions de directives sur lesquelles la section spécialisée du CES s'est déjà prononcée, décembre 1970 . 
Si une première mouture du texte circula dans les ministères dès l'automne 1969, la proposition définitive fut publiée en mai 1970. Celle-ci reprenait les éléments proposés en décembre 1965 par l'Unesem et ceux du codex, mais ne se référait explicitement qu'à ces derniers. La directive proposait de se fonder sur une double définition des eaux minérales naturelles. Les États membres pouvaient, selon leur choix, reprendre dans leur législation soit la définition de type latin, soit la définition de type germanique, soit les deux. La proposition de directive eut un accueil favorable des professionnels de l'Unesem. Elle suivit dès lors le circuit prévu par l'article $100 \mathrm{du}$ traité. Elle fut transmise le 11 mai 1970 au Conseil des Communautés européennes. Celui-ci, lors de la session des 25 et 26 mai 1970, décida de consulter le CES et le Parlement européen. Le CES analysa la proposition dans le cadre de sa section spécialisée pour les questions économiques dont les rapports furent présentés en janvier et mars 1971. Dans le même temps, lors de sa séance du 15 juin 1970, le Parlement renvoya cette proposition de directive aux diverses commissions déjà évoquées. En dehors de quelques remarques sur des problèmes de traduction, et d'absence d'exposé des motifs, elle fut adoptée. ${ }^{24}$ Ainsi, les organes consultatifs avaient-ils accepté les principes et les procédures proposées par le projet. Restait le plus important, la phase ultime d'approbation par le Conseil des ministres et son comité des représentants permanents, c'est-à-dire la véritable phase de négociation.

\section{Une négociation très difficile}

Très rapidement, les négociateurs allemands repoussèrent un texte dont pourtant leur organisation professionnelle, le VDM, avait été un des principaux rédacteurs quelques années plus tôt. ${ }^{25}$ Comment expliquer ce «revirement»? En réalité, le contexte avait changé entre la réalisation des compromis au sein du Gesem (1965) et du Codex (1968) et la phase de négociation effective.

En effet, le processus qui avait abouti au compromis au sein du comité spécialisé des eaux minérales du Codex, était alors soumis au niveau hiérarchique supérieur, avec notamment l'intervention de l'Organisation Mondiale de la Santé. Les experts de l'OMS s'opposèrent à l'adoption d'une définition des eaux minérales comme étant «douées de propriétés favorables à la santé» en 1971. Leur doctrine était simple: aucun produit ne saurait faire état de propriétés relatives à la santé s'il n'était pas un médicament. Les délégués officiels du gouvernement de la RFA, du Royaume-Uni, des Pays-Bas suivirent les délégués de l'OMS et refusèrent de reconnaître ce type de définition pour l'eau minérale. Pour régler le problème, un groupe d'experts fut alors nommé par l'OMS. Mais, constitué uniquement de pharmacologues, il suscita l'ire

24. CHAN, 2003 0384, art.5, DGS, Document de travail sur la proposition de directive, 07.10.1969; UE, BAC 003/1974 - 85, Proposition de directive, document du 24.03.1970 et du 04.05.1970; AHUE, CES 3542, Mathias Berns, Président du CES à Jacques Genton, Secrétaire général, 26.05.1970; Compte rendu des délibérations du CES, 18.03.1971; AHUE, POE 1097, Documents parlementaires. Compte rendus et rapport de la commission des affaires sociales et procès-verbaux des sessions du parlement. Réunions du 29.06., 01.12.1970 et des 27 et 28.01.1971.

25. AHUE, BAC $028 / 1980$ - 588, Résumé aide mémoire, septembre 1973. 
des «Latins» car aucun d'entre eux ne considéraient l'hydrologie médicale ou thermale comme une spécialité digne d'intérêt. Leurs conclusions, rendues en mars 1972, étaient sans appel: non seulement il était impossible de dire que les eaux minérales avaient un effet bénéfique pour la santé, mais un grand nombre d'entre elles pouvaient au contraire avoir un effet défavorable. ${ }^{26}$

Dans le même temps, les rapports de force dans l'industrie des eaux minérales avaient été modifiés depuis les négociations du début des années 1960. Les entreprises allemandes et françaises étaient alors de taille souvent équivalente et la position germanique au sein de la CEE paraissait isolée. L'affirmation des «géants» français au début des années 1970, résultat d'une forte concentration de la branche, était dorénavant de nature à inquiéter les Allemands, restés très faiblement concentrés. En outre, la politique industrielle allemande était désormais attentive à la défense dans le cadre communautaire de secteurs traditionnellement faibles, comme l'industrie alimentaire. ${ }^{27}$ L'élargissement de la Communauté au Royaume-Uni, au Danemark et à l'Irlande joua aussi, en modifiant l'avantage qui existait au sein des pays en faveur du modèle latin de régulation. Ces nouveaux arrivants étaient totalement en phase avec les conclusions de l'OMS. Enfin, l'Unesem était sous présidence allemande pendant la période. Même si Otto Wuttke était l'homme qui avait, avec René Loubet, lancé l'Europe des eaux minérales, il était évident que cette position de force au sein de l'organisation professionnelle n'était pas étrangère au blocage. Enfin, au plan institutionnel européen, il faut rappeler que le Conseil fonctionnait sur le mode du compromis de Luxembourg de 1966. Autrement dit, on ne prenait pas de décision à la majorité sans s'être assuré qu'elle puisse finalement être adoptée à l'unanimité. Le refus allemand s'expliquait donc par un contexte général nouveau. Il fut surtout synonyme de blocage de la proposition de directive.

À partir de 1973, les négociations étaient donc au point mort. Pire, les éléments du compromis initial étaient de plus en plus remis en question. Une controverse éclata ainsi à propos de la teneur en bactéries banales au sein des eaux en bouteille. Pour les uns, les germes quels qu'ils soient n'étaient aucunement des composants de l'eau, mais au contraire des indices de souillure. L'idéal implicite était la stérilité absolue. Cette conception prévalait dans la vision anglo-saxonne de l'eau embouteillée. L'avantage des eaux gazeuses était d'empêcher tout développement des bactéries dans la bouteille grâce à l'effet du CO2. ${ }^{28}$ Pour les autres, les germes saprophytes, exempts de toute contamination extérieure, étaient non seulement inoffensifs, mais, par leur adaptation au milieu, dépourvus de matières organiques, ils constituaient sans doute un élément important de protection de l'eau contre le développement de germes allogènes. Pour ces experts, essentiellement français et italiens, il était irréaliste et injustifié de vouloir faire de l'eau un élément stérile. La présence dans l'eau de ces

26. CHAN 2000 113, art.32, DGS, Rapport confidentiel sur la séance consultative concernant la proposition de standard Européen pour les eaux minérales, octobre 1971 et 12 et 13.03.1972.

27. J-C. DEFRAIGNE, op.cit., p.240.

28. La présence de $\mathrm{CO}^{2}$ dans l'eau rend celle-ci «bactériostatique», c'est-à-dire qu'elle arrête la multiplication des bactéries sans les détruire. La quasi totalité de l'industrie allemande, produisant de l'eau gazeuse, était favorable à cette définition. 
types de germes était sans inconvénient, même s'ils se développent après embouteillage. Le problème venait de ce que certaines eaux minérales embouteillées contenant des germes n'étaient pas considérées comme potables par la presque totalité des législations régulant la consommation de l'eau.

En 1975, le gouvernement allemand, se prévalant du fait que les États pouvaient exercer leur propre responsabilité dans le domaine des règles bactériologiques, arguant aussi du droit d'intervention de ces mêmes États en matière de protection de la santé contre les épidémies, prit une décision unilatérale. Dans le cadre d'une ordonnance sur les eaux de boisson, il décida que les eaux minérales devaient être soumises aux mêmes exigences que les eaux traitées: soit un seuil fixé à 1.000 germes cultivant à 22 degrés par ml. ${ }^{29}$ Cette décision était de nature à interdire toutes les importations d'eaux minérales plates en provenance des pays latins. Ces derniers déposèrent un recours devant la Cour constitutionnelle de Karlsruhe et devant la Commission de la $\mathrm{CEE}$ à qui ils demandèrent de saisir la Cour de justice européenne. Finalement, le président anglais du Coreper, Anthony Kinch, obtint un compromis en proposant que l'ordonnance allemande ne s'appliqua que transitoirement aux eaux minérales en l'absence d'une réglementation communautaire.

\section{Le dénouement 1978-1980}

La $\mathrm{CE}$ tenta à plusieurs reprises de sauver, puis de relancer les négociations. Elle avait créé en 1974 un «comité de concertation» chargé de trouver une solution de compromis au «comité de coordination» du Codex, à l'origine du blocage. De leur côté, les pays «latins» avaient organisé en 1975 un grand colloque international à Rome, pour travailler plus particulièrement sur la bactériologie de l'eau. Les discussions permirent d'aboutir à une entente minimale reconnaissant l'innocuité des bactéries profondes. Plutôt que de fixer un seuil très bas de tolérance de germes banals dans les bouteilles, comme prévu initialement par les experts de l'OMS, l'accord proposait de s'en tenir à ce que la directive CEE et la norme Codex prévoyaient: des contrôles $\mathrm{du}$ «microbisme normal», dépendant donc des caractéristiques des nappes des différentes eaux. Ce compromis fut accepté par le comité de coordination pour l'Europe à Vienne en juin 1977, et en avril 1978 à l'assemblée générale du Codex à Rome. ${ }^{30}$

En 1978, le Conseil des Communautés européennes avait décidé de réunir des groupes ad hoc pour voir comment on pouvait avancer dans certains dossiers relatifs à l'harmonisation des législations, et particulièrement dans le domaine alimentaire. Les services du Conseil - en réalité, un groupe de conseillers et attachés des Représentations permanentes des États membres -, élaborèrent un document de travail qui

29. CHAN, 1987 0334, art.44, Aide mémoire des services de la Commission, octobre 1978; Paul Bordier au Président du Coreper, 02.11.1979.

30. Programme mixte FAO/OMS sur les normes alimentaires, ALINORM 79/38. Rapport de la 13e session de la commission mixte Fao/Oms du codex alimentarius, 3-14 décembre 1979, Rome; ALINORM 79/38, mars 1980, pp.300-302. 
circula au printemps $1978 .{ }^{31}$ Il proposait de bâtir un nouveau compromis pour débloquer la situation, en donnant raison pour partie aux deux camps en présence. Le document de travail précisait bien que

«l'eau minérale est une denrée alimentaire et qu'il est nécessaire pour les règles générales d'étiquetage des denrées alimentaires d'interdire toutes les indications attribuant à ces denrées des propriétés de prévention, de traitement ou de guérison d'une maladie humaine. [Mais] l'eau minérale naturelle se distingue nettement de l'eau ordinaire par sa nature, ses effets et sa pureté originelle qui sont de nature à lui apporter des propriétés favorables à la santé». ${ }^{32}$

Un groupe «denrées alimentaires» entreprit l'examen de la liste des diverses mentions relatives aux propriétés favorables à la santé qui pourraient être autorisées sur le plan communautaire.

Ce processus fut un succès, car la rédaction d'un projet de directive directement négocié par les délégations des États membres reprit, même s'il donna lieu à des tractations très serrées. Chaque point faisait l'objet de discussions, de «réserves» ou d'acceptations. Les passages les plus discutés concernaient les annexes techniques, jugées primordiales par tous les participants. Les indications concernant les composants essentiels (magnésium, chlorure, etc.), le nombre de milligrammes par litre, etc., donnèrent lieu à des controverses byzantines. Les Français, les Italiens et les Belges se concentraient particulièrement sur les indications portées sur les étiquettes, car ils étaient persuadés que c'est l'élément le plus important à préserver. Ils craignaient par-dessus tout que les pays anglo-saxons refusent toute indication d'effets favorables à la santé sur les étiquettes. ${ }^{33}$

Le cas de l'utilisation de l'eau minérale pour l'alimentation des nourrissons est un des exemples des problèmes posés par l'étiquetage. Au cours d'une réunion du début décembre 1978, la délégation allemande suggéra d'introduire la possibilité pour les États membres

«d'interdire ou de soumettre à certaines conditions - tant sur les emballages, les étiquettes que dans les publicités - toute référence au caractère approprié d'une eau minérale naturelle pour l'alimentation des nourrissons». ${ }^{34}$

Cette proposition suscita immédiatement une ferme opposition des «Latins». En effet, des sociétés comme la San Gemini ou Évian, avaient construit toute leur stratégie de communication sur l'alimentation des nourrissons. Pour les négociateurs français ou italiens, s'il apparaissait légitime de soumettre la mention d'une appropriation particulière à l'alimentation des jeunes enfants à certaines exigences - comme le taux de minéralisation ou la teneur en nitrate par exemple - il n'était pas concevable qu'une

31. CHAN 1987 0334, art.44, DGS, Résumé aide mémoire, 08.06.1978; Document de travail des services de la Commission, 28.09.1978.

32. Ibid., Aide mémoire du Conseil, 19.10.1978.

33. CHAN, 1987 0334, art.44. Propositions sur les indications concernant les composants essentiels des eaux minérales, 27.10.1978; Paul Bordier à Marcel Cats et Carmelo Callipo, 23.05.1978.

34. CHAN, 1987 0334, DGS, Résumé aide mémoire des services du Conseil des Communautés européennes, 19.10.1978. 
interdiction puisse être édictée sans motif. Les négociateurs allemands utilisèrent alors les conclusions d'une négociation parallèle, celle sur la directive eaux potables (eaux destinées à la consommation autres que les eaux minérales), qui était elle aussi en cours, pour évoquer le danger des germes dans l'eau. ${ }^{35}$ Une campagne de presse très dure fut effectuée en Allemagne en décembre 1978 et janvier 1979 présentant les eaux minérales françaises et italiennes comme des dangers pour les nourrissons. Selon la presse, citant des experts allemands, l'eau du robinet présentait «beaucoup moins de risques que les eaux minérales françaises et italiennes»». ${ }^{36} \mathrm{~L}$ 'affaire fit grand bruit, et les chancelleries furent même mobilisées pour voir quels pouvaient être les produits allemands susceptibles de faire l'objet de mesures restrictives, en particulier certaines bières importées. Cet épisode provoqua une forte exaspération et suscita néanmoins une mobilisation qui permit d'aboutir au déblocage de la situation.

En effet, les Italiens annoncèrent le blocage de la directive «eau potable» aussi longtemps que les autorités allemandes refuseraient le compromis dans le domaine de la proposition de directive «eaux minérales». Les négociateurs latins jugeaient eux-mêmes que cette tentative «tactique», était la dernière qu'ils avaient à proposer. ${ }^{37}$ Le cours des négociations fut aussi bouleversé par l'adoption d'une jurisprudence de la Cour de justice des Communautés européennes connue sous le nom de jurisprudence «cassis de Dijon». Depuis le début des années 1970, la Cour avait été saisie de nombreuses plaintes dénonçant les obstacles à la libre circulation des produits. Ces plaintes reposaient sur l'article 28 du traité de Rome interdisant des mesures protectionnistes techniques. Elles aboutirent à une décision importante en février 1979. Il s'agissait d'un principe de reconnaissance mutuelle par les États membres de la Communauté, de leurs réglementations respectives en l'absence d'harmonisation. ${ }^{38}$ Cette jurisprudence allait se montrer déterminante dans le déblocage de la situation. Dans leurs commentaires, les organisations professionnelles estimaient que la jurisprudence pouvait tout à fait être appliquée au cas des eaux embouteillées. Pour l'Allemagne, il valait mieux accepter une directive d'harmonisation reposant sur un compromis, alors qu'une absence d'harmonisation aurait, en suivant la jurisprudence

35. CHAN, 1987 0334, art.44. Rapport du Coreper au Conseil, 08.12.1978.

36. Vorsicht vor „Baby-Mineralwasser “, in: Stuttgarter Zeitung, 22.12.1978; Norm für Wasser gesetzt, in: Frankfurter Rundschau, 23.12.1978; Ist ausländisches Mineralwasser eine „Brutstätte für Keime"?, in: Die Welt, 22.12.1978; Stille Wasser: Für Babys oft ungesund, in: Welt am Sonntag, 24.12.1978.

37. CHAN, 1987 0334, art.44, Ambassade de France à Bonn à Paul Bordier, 22.12.1978 et 14.02.1979; Directeur général de la Santé au ministre de la Santé, 06.06.1979.

38. Arrêt de la Cour de justice des Communautés européennes, 20.02.1979. - Rewe-Zentral AG contre Bundesmonopolverwaltung für Branntwein. - Demande de décision préjudicielle: Hessisches Finanzgericht - Allemagne. Affaire 120/78, Recueil de jurisprudence 1979, p.649. Cf. aussi Recueil CJCE, 1979, p.649; J. BOULOUIS, R. CHEVALLIER, Grands arrêts de la Cour de justice des Communautés européennes, tome 2, Dalloz, Paris; A. MATERA, Revue du marché commun, 1980, p.514; E. VALCESCHINI, Entreprises et pouvoirs publics face à la qualité. Les produits agroalimentaires dans le marché européen, in: G. ALLAIRE, R. BOYER (eds), La grande transformation de l'agriculture, lectures conventionnalistes et régulationnistes, INRA - Economica, Paris, 1995, p.66. 
du cassis de Dijon, permis aux produits français, italiens et belges de pénétrer le marché allemand.

Les conditions juridiques étaient ainsi largement modifiées, tandis que le Comité des représentants permanents, considérant comme prioritaire la directive sur les eaux potables, essaya de trouver rapidement des solutions. Cette conjonction d'éléments permit d'aboutir à une entente rapide et à l'adoption en conseil des Ministres des directives 80/777 sur les eaux minérales et 80/778 sur l'eau de consommation humaine. ${ }^{39}$ La rédaction définitive donna une définition complexe de l'eau minérale, associant les critères quantitatifs et qualitatifs. On en revenait globalement au compromis initial, celui de l'organisation professionnelle européenne. Son dispositif principal et ses annexes énonçaient de façon détaillée les prescriptions d'hygiène, de préservation de la qualité et d'étiquetage. La reconnaissance, même strictement encadrée, de propriétés favorables à la santé, la mise au point d'une règle d'autorisation préalable par une autorité médicale compétente, même si la reconnaissance d'une eau minérale par la simple teneur en minéraux n'est pas interdite, pouvait être considérée comme une « victoire » du modèle latin. La directive posait en principe que les eaux minérales n'étant pas des médicaments, elles ne pouvaient pas faire état de propriétés de prévention, de traitement ou de guérison des maladies humaines. Elle laissait aux autorités nationales compétentes le droit d'autoriser les mentions d'effets généraux sur certaines fonctions organiques, telles que «diurétiques, stimulant la digestion, etc.». Là encore, on peut reconnaître les principes défendus par la France, la Belgique et l'Italie.

\section{Application et impact de la directive CE 877}

\section{L'application de la norme dans les États membres}

Les États membres disposaient d'un délai limite de deux ans pour admettre la libre commercialisation des eaux minérales conformes aux prescriptions de la directive et de quatre ans pour interdire celles qui ne le seraient pas. La longueur du délai tenait compte en réalité du temps nécessaire pour mettre en place les procédures d'autorisation là où elles n'existaient pas. L'adaptation s'effectua de manière plus ou moins rapide selon les États. Elle ne posa pas de problème particulier dans les pays du domaine latin. L'Italie, la Belgique, la France, adaptèrent rapidement les prescriptions d'admission de libre circulation des eaux. L'application complète ne fut toutefois organisée réellement en France que plus tardivement, en 1989. L'Allemagne adapta elle aussi sa législation dans des délais assez brefs, en $1984 .{ }^{40}$ La législation «latine»

39. Directive 80/777/CEE du Conseil, 15.07.1980; Directive 80/778/CEE du Conseil, du 15.07.1980.

40. Arrêté sur les conditions d'importation en France des eaux minérales naturelles étrangères, in: Journal Officiel, 24.12.1983, p.11484; Decreto ministeriale del 01.03.1982, nuove norme per le etichette delle acque minerali, in: GURI, Serie generale, 10.02.1983, p.1009; Mineral- und Tafelwasser-Verordnung, 01.08.1984, in: Bundesgesetzblatt IS, p.1036. 
s'imposa peu à peu dans ce pays. On en veut pour preuve le fait que les Heilwässer furent introduites officiellement dans la catégorie des «eaux minérales naturelles» et comptabilisées dans les statistiques après $1994 !^{41}$

Les pays anglo-saxons ne mirent pas en œuvre les procédures de reconnaissance des propriétés favorables à la santé, s'en tenant à la vision germanique des degrés de minéralisation. C'est d'ailleurs ce point qui posait un problème important aux Britanniques. En effet, lors de la préparation en 1983 de l'application de la directive, ils se rendirent compte que certaines de leurs eaux ne correspondaient ni à la définition latine ni à la définition germanique. C'était le cas en particulier, de l'eau de Malvern, une des eaux embouteillées les plus connues du Royaume. «L'eau minérale est intéressante surtout parce qu'elle ne contient rien, parce que c'est juste de l'eau pure», avait pu dire le conservateur Michael Spicer dans la discussion qui eut lieu aux Communes. Selon lui,

«contrairement aux exemples français ou continentaux, il n'a jamais été revendiqué que l'eau de Malvern possédait des vertus quasi médicales ou autres pouvoirs magiques. En effet, elle n'a jamais été vendue que pour ce qu'elle est: de l'eau pure».

L'eau de Malvern étant fournisseur de la famille royale depuis la Reine Victoria, il lui apparaissait «incroyable que ce qui convient à la famille royale ne convienne pas à la Communauté économique européenne». Ces mouvements d'humeur n'empêchèrent pas le Royaume-Uni de mettre au point un texte qui le mettait en conformité avec la directive, en 1985. En 1988, la liste des eaux minérales embouteillées reconnues par la Communauté comptait 19 sources et Malvern Water en faisait partie. Les analyses précises déterminèrent un degré de minéralisation faible, mais bien présent! ${ }^{42}$

D'autres États membres, notamment les Pays-Bas, furent plus réticents. Pendant longtemps, ils n'appliquèrent pas la directive, ce qui n'avait pas une importance majeure car ils n'étaient pas producteurs. En revanche, les États méditerranéens dont la procédure d'entrée au sein de la Communauté était en cours, suivaient de très près les négociations. L'Espagne, par exemple, était en attente depuis longtemps de la législation européenne pour modifier son ancien décret de 1928. Dès que la directive fut connue, le ministère de la Santé espagnol lança la rédaction d'un texte conforme en tous points à la norme $\mathrm{CE} .{ }^{43} \mathrm{C}$ 'est ainsi l'Espagne qui fut la première à adapter sa législation, alors qu'elle ne faisait pas encore partie de la Communauté. La Grèce avait aussi préparé activement la refonte de sa législation. En 1979, le docteur Bernard Ninard, directeur du département des eaux hydrologiques et thermales du Laboratoire

41. H-J. TEUTEBERG, Vom Gesundbrunnen in Kurbädern zur modernen Mineralwasserproduktion, in: R. WALTER (dir.), Geschichte des Konsums: Erträge der 20. Arbeitstagung der Gesellschaft für Sozial- und Wirtschaftsgeschichte, Franz Steiner Verlag, Stuttgart, 2004, pp.123-158, ici p. 125.

42. House of Commons, Deb 15.2.1983 vol.37 cc268-74, resp. 24.02.1988 vol 128 c240W, Malvern Water; The Natural Mineral Waters Regulations, 1985.

43. Decreto Real 3069/1972, 26.10.1972 resp. 211/1981, 24.07.1981, in: Boletín Oficial del Estado, 21.09.1981. 
national français de la Santé y effectua plusieurs missions pour encadrer les responsables administratifs et techniques du contrôle des eaux. ${ }^{44}$

\section{L'impact de la norme}

Les objectifs de la norme CE étaient nombreux et ambitieux. Il s'agissait d'éliminer les entraves règlementaires, d'affirmer la spécificité des eaux minérales par rapport à d'autres catégories de boisson et d'apporter des garanties hygiéniques supplémentaires en généralisant à l'ensemble des exploitations la surveillance permanente des installations d'embouteillage et de la bactériologie qui étaient déjà en pratique dans les plus importantes d'entre elles. Dans tous ces domaines, il est évident que la directive a porté ses fruits. Le nouveau décollage qu'ont connu les eaux embouteillées de la fin des années 1980 jusqu'au début du XXIe siècle, s'explique pour une large part par l'adoption de tous ces critères. De même, la norme devait, selon ses concepteurs, avoir des conséquences bénéfiques pour la protection du consommateur car elle clarifiait, améliorait et authentifiait l'information sur l'origine, la composition et les propriétés vérifiées de chacune de ces eaux par l'étiquetage. Là encore, l'accueil a été très positif car les consommateurs ont adopté les eaux embouteillées massivement, en raison de la confiance que leur inspire ce type de produit.

Toutefois, le but était aussi de créer un marché intégré des eaux minérales européennes et dans ce domaine, il faut bien constater que la directive a été un échec. Il n'existe aucun obstacle à la circulation des produits, mais force est de constater que le marché européen n'est la plupart du temps qu'une juxtaposition de marchés nationaux. Certes, quelques produits circulent dans plusieurs pays comme par exemple, la San Pellegrino. Mais il s'agit là du résultat de stratégies de gamme de grands groupes internationaux. Bien sûr, le taux d'exportation d'un pays comme la France s'élève à $32 \%$ pour les eaux minérales en 1996. Mais le cas français reste exceptionnel. En dehors de quelques zones frontalières, les eaux minérales étrangères ne sont guère présentes dans les grandes surfaces ni chez les autres détaillants. Le marché allemand, par exemple, n'a jamais été conquis par les grands embouteilleurs français. On peut supposer que les polémiques de la fin des années 1970 ont laissé des traces. On peut aussi penser que, pour les consommateurs allemands, l'emballage était un obstacle majeur, du moins jusqu'à l'apparition des grandes bouteilles en PET. Certains conflits sur les marques ont réapparu à l'occasion de la libre circulation instaurée par l'application de l'harmonisation. C'est le cas de l'ancienne lutte entre Vichy Catalàn et les entreprises de Vichy, qui n'acceptent pas que cette eau puisse être importée en France sous ce nom. ${ }^{45}$

Il est nécessaire de dire que la norme européenne a eu un profond impact par la suite, puisque l'essentiel de ses éléments a été repris dans le Codex Alimentarius mondial. L'enjeu de ces normes du code mondial est devenu majeur car elles étaient

44. CHAN, 1987 0384, art.44, Ministère grec de la Santé à Simone Weil, 17.01.1979.

45. Cf. Cour de Cassation, ch. com., arrêt du 16.11.2004, Société Compagnie Fermière de l'Établissement thermal de Vichy c. Entreprise IBB. 
désormais reconnues comme valides par l'OMC et s'imposent donc à tous les opérateurs économiques. ${ }^{46}$ Lors de la réunion de juin 1997 à Genève qui devait proposer la norme eau minérale du Codex, la norme européenne suscita une méfiance maximale des négociateurs américains et asiatiques. Il fallut en arriver à un vote. Le résultat fut très serré: la norme européenne recueillit 33 voix pour et 31 contre, 10 délégations s'abstenant. ${ }^{47}$ Ces normes contraignantes sont sans doute pour beaucoup dans l'extraordinaire croissance qu'a connu à l'échelle mondiale le marché des eaux minérales au cours des années 1990 et 2000.

\section{Conclusion}

La dynamique suscitée par les Communautés européennes aboutit donc de manière positive à une harmonisation. Ce succès n'est pas négligeable si l'on songe que les hygiénistes engagés dans la lutte contre la fraude et l'adultération, n'avaient abouti à aucun résultat lors des premières tentatives de convergence. Les institutions européennes ont donc réussi là où les experts et les hygiénistes d'avant la Première Guerre mondiale avaient échoué. C'est d'autant plus remarquable que dans le cas des eaux embouteillées, les exigences en termes de réglementation étaient très élevées et les divergences entre systèmes assez lourdes. Souvent, pour supprimer les entraves non tarifaires aux échanges, les principes admis par la CEE ont eu tendance à aller dans le sens d'une dérèglementation, ou du moins en limitation de la réglementation aux exigences dites essentielles. ${ }^{48}$ La confrontation des modèles aboutit donc à un compromis qui valide l'essentiel des principes retenus par les pays «latins». Il est clair que cette norme ne s'est pas imposée «naturellement». Le compromis final repose sur une combinaison complexe d'intérêts étatiques, de rapports de forces industriels et de visions divergentes d'experts. ${ }^{49} \mathrm{Il}$ est certain que la norme $\mathrm{CE}$ a été en grande partie une des raisons du succès du produit «eau minérale» dans les années qui ont suivi. Pour les consommateurs, les institutions impliquées dans le système de régulation inspiraient une grande confiance dans le produit «eau minérale» et plus largement, pour l'eau embouteillée.

46. Norme Codex Stan 108-1981.

47. Les pays qui ont voté pour la norme européenne sont les 15 pays de l'UE, le Burundi, le Cameroun, la Côte d'Ivoire, la Croatie, Chypre, l'Estonie, la Hongrie, le Mozambique, la Norvège, le Pérou, la Pologne, la Roumanie, la Slovaquie, la Suisse, la Tunisie, la Turquie, l’Uruguay.

48. F. NICOLAS, E. VALCESCHINI, La dynamique économique de la qualité agro-alimentaire, in: F. NICOLAS, E. VALCESCHINI (éds), Agroalimentaire. Une économie de la qualité, Economica, Paris, 1995, p.24.

49. À titre de comparaison, il peut être utile de voir que dans d'autres domaines, l'émergence de standards est toujours douloureuse et repose aussi sur la confrontation et le rapport de force. Cf. M. MOGUEN TOURSEL, Emergence and transfer of vehicle safety standards: why we still do not have global standards, in: Entreprises et Histoire, juin 2008, pp.88-102. 